\title{
手性联萗酚聚合物在不对称环氧化反应中的应用
}

\author{
蔡瀚欧阳昆冰*阳年发* \\ (湘潭大学化学学院 环境友好化学与应用省部共建教育部重点实验室＼cjkstart湘潭４11105)
}

\begin{abstract}
摘要 小分子手性催化剂存在着不便于与产物分离且催化剂难以回收重复使用等问题, 这使得聚合物负载型的可回收 催化剂得到了广泛的关注和研究. 本工作成功地将可重复使用的手性联䒺酚聚合物负载的二乙基锌催化剂用于 $(E)-\alpha, \beta-$ 不饱和芳基酮的不对称环氧化反应中. 在对反应条件进行了优化后研究了反应的底物普适性, 进一步探索了回收后的 催化剂在以上不对称反应中的催化效率. 该反应中对映体过量值最高能达到 $94 \%$. 分别对聚合物进行了回收利用实验, 结果表明经过多次重复使用的聚[(S)-3-丙烯酰胺基-2,2'-二垟基-1,1'-联二荎]的手性诱导能力并没有明显下降.
\end{abstract}

关键词 联䒺酚; 手性聚合物; 二乙基锌; 查尔酮; 不对称催化

\section{Application of Chiral 1,1'-Bi-2-naphthol Polymers in Asymmetric Epoxidation of $(E)-\alpha, \beta$-Unsaturated Aryl Ketones}

\author{
Cai, Han Ouyang, Kunbing* Yang, Nianfa* \\ (Key Laboratory of Environmentally Friendly Chemistry and Applications of Ministry of Education, College of Chemistry, \\ Xiangtan University, Xiangtan 411105)
}

\begin{abstract}
Most small chiral molecule catalysts are suffered from a rigid process in both product separation and recovery. Therefore more attention has been drawn to the soluble polymer-supported catalyst which could be easily recycled. In this paper, a new type of reusable chiral binaphthol polymer-supported diethylzinc catalyst was synthesized and applied in the asymmetric epoxidation of $(E)-\alpha, \beta$-unsaturated aryl ketones. The scope of this reaction was explored. Various $(E)-\alpha, \beta$-aryl ketones could be easily prepared in good yield (up to $88 \%$ ) and high $e e$ value (up to $94 \%$ ) via this asymmetric epoxidation process. Ligands were recovered to explore the inductive effect of the reaction. Recovery experiments of this binaphthol polymer-supported diethylzinc catalyst were conducted. The results indicate that the asymmetric induction ability of the reclaimed chiral polymer 1,1'-bi-2-naphthol did not decrease significantly.
\end{abstract}

Keywords 1,1'-bi-2-naphthol, Et ${ }_{2} \mathrm{Zn}$, chiral polymer, asymmetric addition, aromatic ketone

$\alpha, \beta$-不饱和芳基酮的不对称环氧化反应是合成具有 光学活性 $\alpha, \beta$-环氧芳基酮的重要方法之一 ${ }^{[1]}$. 目前催化 此类反应的主要催化剂为金属有机催化剂 $[2,3]$, 相转移 催化剂 ${ }^{[4]}$ 以及多肽催化剂 ${ }^{[5]}$. 其中金属催化剂应用的较 多且其相关研究也较为成熟. 然而, 金属有机催化剂受 溶剂和其他催化微环境因素的影响较大, 且需要价格较 相对昂贵并有一定的毒性的过渡金属 ${ }^{[2,6]}$. 小分子催化 不对称环氧化反应也有了一定的进步与发展 ${ }^{[2,7,8]}$, 但是 小分子手性催化剂存在着产物不便于分离且催化剂难 以回收重复使用等问题. 聚合物催化此类反应的研究较 少. 相对而言, 聚合物催化此类反应的产物易于分离,
对环境的影响较小, 只需通过简单的过滤洗涤就能进行 重复的回收利用等优势，使其成为当前不对称催化的一 个热点 ${ }^{[9]}$. 将手性联芸酚聚合物应用在不对称催化反应 中也是研究的热点之一 ${ }^{[10,11]}$.

本课题组 ${ }^{[11]}$ 曾研究过聚 $[(S)$-3-丙烯酰胺基-2,2'-二 羟 基-1,1'-联二萗]的合成, 并将其用于催化二乙基锌与 芳香醛的不对称加成反应中. 此聚合物的合成路线如 Scheme 1 所示. 在本课题组研究基础上, 本文将可重复 使用的手性联萗酚的聚合物负载的二乙基锌催化剂用 于 $(E)-\alpha, \beta$-不饱和芳基酩的不对称环氧化反应中. 本文 在对反应条件进行了优化后研究了反应的底物普适性,

\footnotetext{
* Corresponding authors. E-mail: kbouyang@xtu.edu.cn; nfyang@xtu.edu.cn Received October 24, 2018; revised January 16, 2019 published March 8, 2019.

Project supported by the National Natural Science Foundation of China (No. 21602188), the Education Department of Hunan Province (No. 17C1524) and the Doctoral Research Initiation Fund of Xiangtan University (No. 15QDZ52).

国家自然科学基金(No. 21602188)、湖南省教育厅一般项目(No. 17C1524)、湘潭大学博士科研启动基金(No. 15QDZ52)资助项目.
} 
并进一步探索了回收后的催化剂在以上不对称反应中 的催化效率.

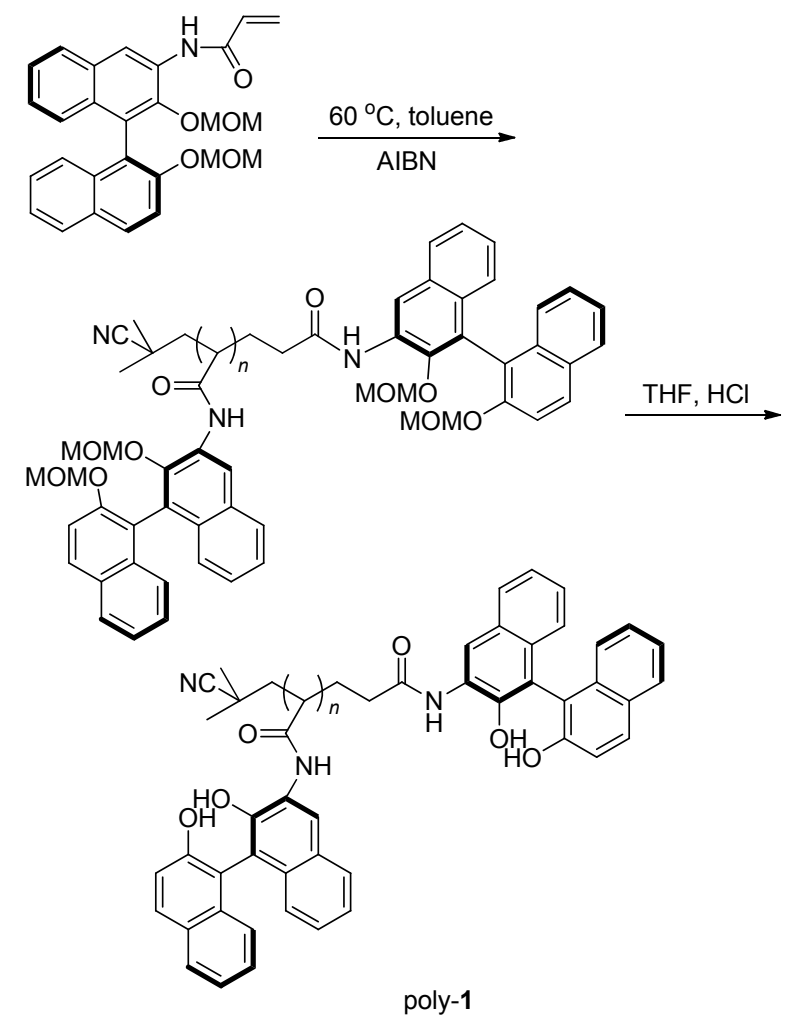

图式 1 聚合物 -1 的合成路线

Scheme 1 Synthetic route of poly-1

\section{1 结果与讨论}

首先合成了聚 $[(S)$-3-丙烯酰胺基-2,2'-二羟基- 1,1'联 二菜 $](\text { poly-1 })^{[11]}$. 随后使用查尔酮作为底物, 用过氧化 氢异丙苯作为氧化剂, 对 $\alpha, \beta$-不饱和芳基酮的不对称环 氧化反应进行了反应条件探索. 如表 1 所示, 考察了溶 剂种类、反应温度、配体及二乙基锌的相对用量对该不 对称环氧化反应的影响. 由表 1 可以看出, 不同的溶剂 对不对称反应有不同的结果, 其中四氢呋喃作为溶剂时 反应结果最好(表 1, Entries 1 4). 在其他反应条件相同 时, 四氢呋喃作为溶剂时得到的产物 $e e$ 值为 $60 \%$, 产率 为 $92 \%$. 这可能是由于四氢呋喃能够促进配体与锌试剂 的配位, 从而提高催化剂对映选择性. 不同的反应温度 对催化效果也有不同的影响, 反应温度越低, 产物的对 映选择性越好(表 1, Entries 2, 5 7). 但将温度降低至零 度以下后, 反应的 $e e$ 值提升并不是很大但产率急剧下 降. 因此, 将温度设置在零度继续对反应进行优化. 配 体 poly-1 与二乙基锌的配比同样对反应结果影响很大 (表 1, Entries 2,8 10), 氧化产物的 $e e$ 值随着 poly-1/Zn 配比的增加有个先升高后下降的过程; 当 poly-1/Zn 的 配比为 $1: 1.4$ 时, 反应产率高达 $95 \%$, 但此时 $e e$ 值却仅
仅为 $60 \%$; 而当 poly-1/Zn 的配比为 $1: 1.2$ 时, 产率与 $e e$ 值都相对较好, 因此选择合适的 poly-1/Zn 的配比为 $1: 1.2$. 然后, 将聚合物的用量的从 $5 \mathrm{~mol} \%$ 增至 15 $\mathrm{mol} \%$, 产率由 70\%增至 88\% (Entries 5, 11 12). 与此同 时, 反应的对映选择性有一个先上升后降低的过程. 最 后考察二乙基锌与 poly-1 在催化过程中所起的作用. 当 二乙基锌的用量为 $12 \mathrm{~mol} \%$, poly-1 的用量为 0 时，氧化 产物外消旋, 说明 poly-1 在不对称催化中对对映选择性 起着重要作用. 当二乙基锌的用量为 0 , 而 poly-1 的用 量为 $10 \mathrm{~mol} \%$ 时, 反应无环氧化产物, 说明在催化过程 中二乙基锌是必须的. 因此我们用异丙醇镧和三乙基铝 代替二乙基锌催化该反应, 发现不管有无 poly-1, 环氧 产物都外消旋(表 1 , Entries $13 \sim 17)^{[8]}$, 因此我们认为二 乙基锌是 Lewis 酸, 活化了不饱和酮, 但同时该不对称 催化也需要合适的 Lewis 酸. 综合考虑上述因素, 最终 选定的最优条件为, poly-1 (10 mol\%), $\mathrm{Et}_{2} \mathrm{Zn}(12 \mathrm{~mol} \%)$, 过氧化氢异丙苯(CMHP) 氧化剂(1.1 equiv.), 反应温度 为 $0{ }^{\circ} \mathrm{C}$, 溶剂为四氢呋喃.

表 1 反应条件的优化

Table 1 Optimization of the reaction condition.

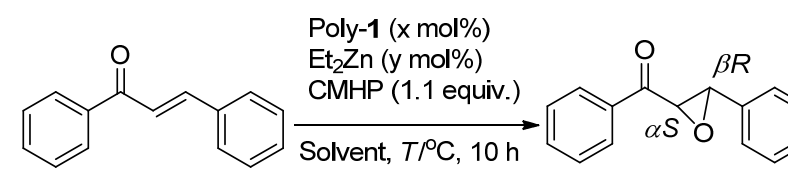

\begin{tabular}{|c|c|c|c|c|c|c|}
\hline Entry & Solvent & $\begin{array}{c}\text { Poly-1 }{ }^{a} / \\
\text { mol\% }\end{array}$ & $\begin{array}{c}\text { Catalyst }^{b} \\
\left(\mathrm{~mol}^{\circ} \mathrm{o}\right)\end{array}$ & $T /{ }^{\circ} \mathrm{C}$ & Yield/\% & $e e^{c} / \%$ \\
\hline 1 & $\mathrm{Et}_{2} \mathrm{O}$ & 10 & $\mathrm{Et}_{2} \mathrm{Zn}(12)$ & 25 & 90 & 48 \\
\hline 2 & THF & 10 & $\mathrm{Et}_{2} \mathrm{Zn}(12)$ & 25 & 92 & 60 \\
\hline 3 & $\mathrm{CH}_{2} \mathrm{Cl}_{2}$ & 10 & $\mathrm{Et}_{2} \mathrm{Zn}(12)$ & 25 & 92 & 55 \\
\hline 4 & Toluene & 10 & $\mathrm{Et}_{2} \mathrm{Zn}(12)$ & 25 & 95 & 50 \\
\hline 5 & THF & 10 & $\mathrm{Et}_{2} \mathrm{Zn}(12)$ & 0 & 85 & 80 \\
\hline 6 & THF & 10 & $\mathrm{Et}_{2} \mathrm{Zn}(12)$ & -25 & 55 & 81 \\
\hline 7 & THF & 10 & $\mathrm{Et}_{2} \mathrm{Zn}(12)$ & -75 & 20 & 83 \\
\hline 8 & THF & 10 & $\mathrm{Et}_{2} \mathrm{Zn}(8)$ & 0 & 70 & 68 \\
\hline 9 & THF & 10 & $\mathrm{Et}_{2} \mathrm{Zn}(10)$ & 0 & 85 & 70 \\
\hline 10 & THF & 10 & $\mathrm{Et}_{2} \mathrm{Zn}(14)$ & 0 & 95 & 60 \\
\hline 11 & THF & 5 & $\mathrm{Et}_{2} \mathrm{Zn}(6)$ & 0 & 70 & 76 \\
\hline 12 & THF & 15 & $\mathrm{Et}_{2} \mathrm{Zn}(18)$ & 0 & 88 & 78 \\
\hline 13 & THF & 0 & $\mathrm{Et}_{2} \mathrm{Zn}(12)$ & 0 & 90 & 0 \\
\hline 14 & THF & 10 & - & 0 & 0 & 0 \\
\hline 15 & THF & 0 & $\left(\mathrm{C}_{3} \mathrm{H}_{7} \mathrm{O}\right)_{3} \mathrm{La}(10)$ & 0 & 92 & 0 \\
\hline 16 & THF & 10 & $\left(\mathrm{C}_{3} \mathrm{H}_{7} \mathrm{O}\right)_{3} \mathrm{La}(10)$ & 0 & 89 & 0 \\
\hline 17 & THF & 0 & $\mathrm{Et}_{3} \mathrm{Al}(10)$ & 0 & 80 & 0 \\
\hline
\end{tabular}

${ }^{\mathrm{c}}$ Determined by HPLC using a Chiralcel AD-H column.

底物拓展实验的结果如表 2 所示. 由表 2 可以看出, 底物为不同的查尔酮衍生物时, 不管苯环上取代基是吸 电子基(表 2, Entries 2 4, 8 9), 还是给电子基(表 2, Entries $5 \sim 6$ ), 反应的产率( $72 \% \sim 88 \%$ ) 和对映选择性 
(79\% 90\%ee) 都有较好的结果. 而当 $\mathrm{R}^{2}$ 取代基为脂肪 族时, 也有较为优秀的产率(Entry 10,83\%)和较为优秀 的对映选择性 $(88 \% e e)$. 在该反应中, 当 $\mathrm{R}^{1}$ 为 2 -荎的查 尔酮的催化反应的立体选择性最好, 其催化产物产率为 $88 \%, e e$ 值为 94\% (表 2, Entry 7).

表 2 poly-1 的锌配合物催化的各种烯酮与 CMHP 的不对称环 氧化反应

Table 2 Asymmetric epoxidation of various enone with CMHP catalyzed by zinc complex of poly-1

\begin{tabular}{|c|c|c|c|c|}
\hline & $\mathrm{R}^{2}$ & $\begin{array}{l}\text { Poly-1 (10 mol\%) } \\
\text { Et }_{2} Z n \text { (12 mol\%) } \\
\text { CMHP (1.1 equiv.) }\end{array}$ & 인 & \\
\hline Entry $^{a}$ & $\mathrm{R}^{1}$ & $\mathrm{R}^{2}$ & Yield $^{b} / \%$ & $e e^{c} / \%$ \\
\hline 1 & $\mathrm{Ph}$ & $\mathrm{Ph}$ & 85 & 80 \\
\hline 2 & $\mathrm{Ph}$ & $4-\mathrm{FC}_{6} \mathrm{H}_{4}$ & 72 & 79 \\
\hline 3 & $\mathrm{Ph}$ & 4- $\mathrm{BrC}_{6} \mathrm{H}_{4}$ & 83 & 89 \\
\hline 4 & $\mathrm{Ph}$ & 4- $\mathrm{ClC}_{6} \mathrm{H}_{4}$ & 88 & 81 \\
\hline 5 & $\mathrm{Ph}$ & $4-\mathrm{OMeC}_{6} \mathrm{H}_{4}$ & 74 & 90 \\
\hline 6 & 4- $\mathrm{OMeC}_{6} \mathrm{H}_{4}$ & $\mathrm{Ph}$ & 85 & 83 \\
\hline 7 & 2-Naphthyl & $\mathrm{Ph}$ & 88 & 94 \\
\hline 8 & $4-\mathrm{NO}_{2} \mathrm{C}_{6} \mathrm{H}_{4}$ & $\mathrm{Ph}$ & 86 & 83 \\
\hline 9 & 4- $\mathrm{ClC}_{6} \mathrm{H}_{4}$ & $4-\mathrm{ClC}_{6} \mathrm{H}_{4}$ & 81 & 82 \\
\hline 10 & $\mathrm{Ph}$ & $t-\mathrm{Bu}$ & 83 & 88 \\
\hline
\end{tabular}

${ }^{a}$ Reactions were carried at $0{ }^{\circ} \mathrm{C}$ for $10 \mathrm{~h}$. The amount of poly-1 was based on a BINOL monomer. ${ }^{b}$ Isolated yield. ${ }^{c}$ Determined by HPLC using a Chiralcel AD-H column. ${ }^{d}$ The absolute configures were determined by comparing with the reported literature. ${ }^{[4,5,13,14]}$

在之前的研究中我们证实了催化剂 poly-1 能回收 并继续用于诱导芳香醛与二乙基锌的不对称催化反应, 而且效果很好. 因此, 我们对本反应中使用的 poly-1 进 行了回收再利用实验研究.

首先, 将催化反应完后的滤渣 $2 \mathrm{~g}$ 溶于 $20 \mathrm{~mL}$ 四氢 呋喃中并滴入盐酸 $(2 \mathrm{~mL})$, 过滤除去不溶物后将滤液滴 入甲醇 $(100 \mathrm{~mL})$ 中. 在析出固体后进行抽滤, 将滤渣用 甲醇洗涤两次, 随后在 $40{ }^{\circ} \mathrm{C}$ 下真空干燥 $8 \mathrm{~h}$ 即得回收 聚合物. 回收的聚合物直接用于不对称催化中. 催化剂 回收再利用的实验结果如表 3 所示. 由表 3 可以看出, 该催化剂虽然可以较容易地回收, 但再利用催化活性和 对映选择性均有所下降, 循环利用 4 次其催化产率从 $85 \%$ 下降到 $65 \%$, 产物 $e e$ 值从 $80 \%$ 下降到 $72 \%$. 由此可

表 3 聚合物-1 的回收利用 ${ }^{a}$

Table 3 Recycled and reused of poly-1

\begin{tabular}{ccc}
\hline Cycle & Yield $^{b} / \%$ & $e e^{c} / \%$ \\
\hline 1 & 85 & 80 \\
2 & 76 & 76 \\
3 & 70 & 75 \\
4 & 65 & 72 \\
\hline
\end{tabular}

${ }^{a}$ Reaction condition: $10 \mathrm{~mol} \%$ recycled poly-1 (based on the repeating unit), 1 mmol of enone, $0.16 \mathrm{~mL}$ of CMHP , $3 \mathrm{~mL}$ of THF carried out at $0{ }^{\circ} \mathrm{C}$ for $5 \mathrm{~h}$.

${ }^{b}$ Isolated yield. ${ }^{c}$ Determined by HPLC using a Chiralcel AD-H column.
以看出，我们研究的手性聚合物有着产物便于分离且催 化剂易于回收重复使用的优势.

\section{2 结论}

将聚 $\left[(S)\right.$-3-丙烯酰胺基- $2,2^{\prime}$-二羟基-1, $1^{\prime}$-联二䒺 $]$ 与 二乙基锌螯合制成了一个手性自负载有机锌试剂，应用 于催化查尔酮及其衍生物的不对称烯烃环氧化的反应. 氧化产物具有中等及良好的产率, 以及良好的对映选择 性. 此外, 催化反应过程操作简单, 自负载锌催化剂仅 通过过滤洗涤过程就能实现回收，且可被循环利用多 次，其回收利用可达到 4 次仍有较好的效果.

\section{3 实验部分}

\section{1 仪器与试剂}

除非另有说明, 所有试剂是购买后未经处理直接使 用的. 实验中使用的四氢呋喃和甲苯是在氮气氛围下使 用钠干燥后蒸馏后得到的. ${ }^{1} \mathrm{H}$ NMR 光谱是在 Bruker $\mathrm{ARX} 400 \mathrm{MHz}$ 光谱仪上测量得到(使用四甲基硅烷 (TMS) 作为内标).

\section{2 实验方法}

在 $0{ }^{\circ} \mathrm{C}$ 下将二乙基锌 $\left(\mathrm{Et}_{2} \mathrm{Zn}\right)(0.12 \mathrm{~mL}, 0.3 \mathrm{mmol})$ 加 入到 $3 \mathrm{~mL}$ poly-1 的四氢呋喃溶液中, 并在室温下氩气 气氛中搅拌 $30 \mathrm{~min}$. 然后, 加入烯酮 $(1 \mathrm{mmol}$ 于 $2 \mathrm{~mL}$ 四 氢呋喃中)并再搅拌 $15 \mathrm{~min}$, 用注射器引入 CMHP (0.16 $\mathrm{mL}, 1.1 \mathrm{mmol})$, 反应混合物在相同温度下进行 $10 \mathrm{~h}$. 将 反应混合物用 $2 \mathrm{~mL}$ 饱和 $\mathrm{NH}_{4} \mathrm{Cl}$ 溶液猝灭, 用 $\mathrm{Et}_{2} \mathrm{O}$ 萃取. 将合并的有机层用 $\mathrm{MgSO}_{4}$ 干燥, 过滤并浓缩至无溶剂. 通过硅胶柱色谱法纯化 $[V$ (石油醚) : $V$ (乙酸乙酯 $)=$ $25: 1]$, 得到环氧化产物, 使用 Chiralcel AD-H 柱通过 HPLC 测定 $e e$ 值.

苯基(3-苯基环氧乙烷-2-基)甲酮(1) ${ }^{[4]}$ : 产率 $85 \%$, $80 \% e e,[\alpha]_{\mathrm{D}}^{25}-165.5\left(c=1.0, \mathrm{CH}_{2} \mathrm{Cl}_{2}\right)[V(n$-hex- ane $)$ : $V($ isopropanol $)=95: 5,1.0 \mathrm{~mL} / \mathrm{min}, t_{1}=18.86 \mathrm{~min}, t_{2}=$ $19.87 \mathrm{~min}] ;{ }^{1} \mathrm{H}$ NMR $\delta: 8.01(\mathrm{~d}, J=4.0 \mathrm{~Hz}, 2 \mathrm{H}), 7.63(\mathrm{t}$, $J=4.0 \mathrm{~Hz}, 1 \mathrm{H}), 7.52 \sim 7.48(\mathrm{~m}, 2 \mathrm{H}), 7.44 \sim 7.26(\mathrm{~m}, 5 \mathrm{H})$, $4.31(\mathrm{~d}, J=4.0 \mathrm{~Hz}, 1 \mathrm{H}), 4.09(\mathrm{~d}, J=4.0 \mathrm{~Hz}, 1 \mathrm{H})$.

苯基(3-(4-氟苯基)环氧乙烷-2-基)甲酮 (2) ${ }^{[13]}$ : 产率 $72 \%, 79 \% e e,[\alpha]_{\mathrm{D}}^{25}-138.5\left(c=1.0, \mathrm{CH}_{2} \mathrm{Cl}_{2}\right)[V(n$-hexane) $: V($ isopropanol $)=95: 5,1.0 \mathrm{~mL} / \mathrm{min}, t_{1}=20.39 \mathrm{~min}$, $\left.t_{2}=22.32 \mathrm{~min}\right] ;{ }^{1} \mathrm{H}$ NMR $\delta: 8.02(\mathrm{~d}, J=8.0 \mathrm{~Hz}, 1 \mathrm{H})$, $7.53 \sim 7.47(\mathrm{~m}, 2 \mathrm{H}), 7.41 \sim 7.26(\mathrm{~m}, 5 \mathrm{H}), 7.10(\mathrm{t}, J=4.0$ $\mathrm{Hz}, 2 \mathrm{H}), 4.28$ (s, 1H), 4.07 (s, 1H).

苯基(3-(4-溴苯基)环氧乙烷-2-基)甲酮 $(3)^{[13]}$ : 产率 $83 \%, 89 \% e e,[\alpha]_{\mathrm{D}}^{25}-135.2\left(c=1.2, \mathrm{CH}_{2} \mathrm{Cl}_{2}\right)[V(n$-hexane) $: V($ isopropanol $)=95: 5,1.0 \mathrm{~mL} / \mathrm{min}, t_{1}=14.06 \mathrm{~min}$, 
$\left.t_{2}=16.69 \mathrm{~min}\right] ;{ }^{1} \mathrm{H}$ NMR $\delta: 8.01(\mathrm{~d}, J=8.0 \mathrm{~Hz}, 2 \mathrm{H})$, $7.65 \sim 7.62(\mathrm{t}, J=7.2 \mathrm{~Hz}, 1 \mathrm{H}), 7.55 \sim 7.47(\mathrm{~m}, 5 \mathrm{H}), 7.27 \sim$ $7.24(\mathrm{~d}, J=11.0 \mathrm{~Hz}, 1 \mathrm{H}), 4.26(\mathrm{~s}, 1 \mathrm{H}), 4.06(\mathrm{~s}, 1 \mathrm{H})$.

苯基(3-(4-氯苯基)环氧乙烷-2-基)甲酮(4) ${ }^{[13]}$ : 产率 $88 \%, 81 \% e e,[\alpha]_{\mathrm{D}}^{25}-195.5\left(c=1.0, \mathrm{CH}_{2} \mathrm{Cl}_{2}\right)[V(n$-hexane) $: V$ (isopropanol $)=95: 5,1.0 \mathrm{~mL} / \mathrm{min}, t_{1}=20.65 \mathrm{~min}$, $\left.t_{2}=24.53 \mathrm{~min}\right] ;{ }^{1} \mathrm{H}$ NMR $\delta: 8.01(\mathrm{~d}, J=7.4 \mathrm{~Hz}, 2 \mathrm{H}), 7.62$ (d, $J=6.8 \mathrm{~Hz}, 1 \mathrm{H}), 7.52 \sim 7.49(\mathrm{t}, J=9.4 \mathrm{~Hz}, 2 \mathrm{H}), 7.40 \sim$ $7.26(\mathrm{~m}, 4 \mathrm{H}), 4.26(\mathrm{~s}, 1 \mathrm{H}), 4.06(\mathrm{~s}, 1 \mathrm{H})$.

苯基(3-(4-甲氧基苯基)环氧乙烷-2-基)甲酮 $(\mathbf{5})^{[13]}$ : 产率 74\%, 90\%ee, $[\alpha]_{\mathrm{D}}^{25}-185.5\left(c=1.0, \mathrm{CH}_{2} \mathrm{Cl}_{2}\right)[V(n-$ hexane) $: V$ (isopropanol) $=95: 5,1.0 \mathrm{~mL} / \mathrm{min}, t_{1}=26.68$ $\left.\min , t_{2}=29.40 \mathrm{~min}\right] ;{ }^{1} \mathrm{H}$ NMR $\delta: 8.03(\mathrm{~d}, J=10.4 \mathrm{~Hz}, 2 \mathrm{H})$, 7.38 (d, $J=7.4 \mathrm{~Hz}, 5 \mathrm{H}), 6.97$ (d, $J=12.4 \mathrm{~Hz}, 2 \mathrm{H}), 4.25$ (s, 1H), 4.07 (s, 1H), 3.88 (s, 3H).

4-甲氧基苯基(3-苯基环氧乙烷-2-基)甲酮(6) ${ }^{[14]}$ : 产 率 $85 \%, 83 \% e e,[\alpha]_{\mathrm{D}}^{25}-171.8\left(c=1.0, \mathrm{CH}_{2} \mathrm{Cl}_{2}\right)[V(n-$ hexane $): V($ isopropanol $)=95: 5,1.0 \mathrm{~mL} / \mathrm{min}, t_{1}=46.65$ $\left.\min , t_{2}=52.53 \mathrm{~min}\right] ;{ }^{1} \mathrm{H}$ NMR $\delta: 8.04(\mathrm{~d}, J=11.8 \mathrm{~Hz}, 2 \mathrm{H})$, 7.40 (d, $J=4.0 \mathrm{~Hz}, 5 \mathrm{H}), 6.89$ (d, $J=12.0 \mathrm{~Hz}, 2 \mathrm{H}), 4.27$ (s, $1 \mathrm{H}), 4.08(\mathrm{~s}, 1 \mathrm{H}), 3.89(\mathrm{~s}, 3 \mathrm{H})$.

4-(2-菜基)-(3-苯基环氧乙烷-2-基)甲酮(7) ${ }^{[15]}$ : 产率 $88 \%, 94 \% e e,[\alpha]_{\mathrm{D}}^{25}-146.9\left(c=1.0, \mathrm{CH}_{2} \mathrm{Cl}_{2}\right)[V(n$-hexane) $: V($ isopropanol $)=99: 1,1.0 \mathrm{~mL} / \mathrm{min}, t_{1}=18.23 \mathrm{~min}$, $\left.t_{2}=19.40 \mathrm{~min}\right] ;{ }^{1} \mathrm{H}$ NMR $\delta: 8.03(\mathrm{~d}, J=7.0 \mathrm{~Hz}, 2 \mathrm{H})$, $7.90 \sim 7.80(\mathrm{~m}, 4 \mathrm{H}), 7.62(\mathrm{~d}, J=6.7 \mathrm{~Hz}, 1 \mathrm{H}), 7.55 \sim 7.45$ (m, 4H), $7.43(\mathrm{~d}, J=8.0 \mathrm{~Hz}, 1 \mathrm{H})$.

4-硝基苯基(3-苯基环氧乙烷-2-基)甲酮 $(\mathbf{8})^{[15]}$ : 产率 $86 \%, 83 \% e e,[\alpha]_{\mathrm{D}}^{25}-102.8\left(c=1.0, \mathrm{CH}_{2} \mathrm{Cl}_{2}\right)[V(n$-hexane) $: V($ isopropanol $)=95: 5,1.0 \mathrm{~mL} / \mathrm{min}, t_{1}=15.42 \mathrm{~min}$, $\left.t_{2}=18.20 \mathrm{~min}\right] ;{ }^{1} \mathrm{H}$ NMR $\delta: 7.88(\mathrm{~d}, J=8.2 \mathrm{~Hz}, 2 \mathrm{H}), 7.65$ (d, $J=7.2 \mathrm{~Hz}, 2 \mathrm{H}), 7.31 \sim 7.43$ (m, 5H), 4.25 (s, 1H), 4.09 (s, 1H);

4-氯苯基(3-4-氯苯基环氧乙烷-2-基)甲酮 $(9)^{[13]}$ : 产 率 $81 \%, 82 \% e e,[\alpha]_{\mathrm{D}}^{25}-125.5\left(c=1.0, \mathrm{CH}_{2} \mathrm{Cl}_{2}\right)[V(n-$ hexane) $: V($ isopropanol $)=95: 5,1.0 \mathrm{~mL} / \mathrm{min}, t_{1}=24.38$ $\left.\min , t_{2}=28.13 \mathrm{~min}\right] ;{ }^{1} \mathrm{H}$ NMR $\delta: 7.92(\mathrm{~d}, \quad J=9.0 \mathrm{~Hz}$, 2H), 7.49 (d, $J=7.4 \mathrm{~Hz}, 2 \mathrm{H}), 7.38(\mathrm{~d}, J=9.4 \mathrm{~Hz}, 2 \mathrm{H})$, $7.31 \sim 7.27$ (t, $J=7.8 \mathrm{~Hz}, 2 \mathrm{H}), 4.20(\mathrm{~s}, 1 \mathrm{H}), 4.06(\mathrm{~s}, 1 \mathrm{H})$;

苯基((3-4-正丁基环氧乙烷-2-基)甲酮 (10) ${ }^{[14]}$ : 产率 $83 \%, 88 \% e e,[\alpha]_{\mathrm{D}}^{25}+35.1\left(c=2.0, \mathrm{CH}_{2} \mathrm{Cl}_{2}\right)[V(n$-hexane) $: V($ isopropanol $)=99: 1,1.0 \mathrm{~mL} / \mathrm{min}, t_{1}=20.39 \mathrm{~min}$, $\left.t_{2}=22.32 \mathrm{~min}\right] ;{ }^{1} \mathrm{H}$ NMR $\delta: 8.00(\mathrm{~d}, J=7.4 \mathrm{~Hz}, 2 \mathrm{H}), 7.63$ (t, $J=7.4 \mathrm{~Hz} 1 \mathrm{H}), 7.54 \sim 7.49$ (t, $J=7.4 \mathrm{~Hz}, 2 \mathrm{H}), 4.15$ (s, 1H), 2.97 (s, 1H), $1.04(\mathrm{~s}, 9 \mathrm{H})$.

辅助材料(Supporting Information) 产物 $1 \sim 10$ 的 ${ }^{1} \mathrm{H}$ NMR 谱图及液相图谱. 这些材料可以免费从本刊网站 (http://sioc-journal.cn/)上下载.

\section{References}

[1] Lauret, C. Tetrahedron: Asymmetry 2001, 12, 2359.

[2] Bougauchi, M.; Watanabe, S.; Arai, T.; Sasai, H.; Shibasaki, M. J. Am. Chem. Soc. 1997, 119, 2329.

[3] Wang, X. W.; Shi, L.; Li, M. X.; Ding, K. L. Angew. Chem., Int. Ed. 2005, 117, 6520 .

[4] Ooi, T.; Ohara, D.; Tamura, M.; Maruoka, K. J. Am. Chem. Soc. 2004, 126, 6844.

[5] Yi, H.; Zou, G.; Li, Q.; Chen, Q.; Tanga, J.; He, M. Y. Tetrahedron Lett. 2005, 46, 5665.

[6] Daikai, K.; Kamaura, M.; Inanaga, J. Tetrahedron Lett. 1998, 39, 7321.

[7] Lattanzi, A. Adv. Synth. Catal. 2006, 348, 339.

[8] Minatti A.; Dötz K. H. Eur. J. Org. Chem. 2006, 1, 268.

[9] (a) Liu. D. C.; Ouyang. K. B.; Yang. N. F. Tetrahedron 2016, 72, 1018.

(b) Zhang. A. L.; Yu. Z. D.; Yang. L. W.; Yang. N. F. Tetrahedron: Asymmetry 2015, 26, 173.

(c) Zhang. A. L.; Yu. Z. D.; Yang. L. W.; Yang. N. F.; Peng. D. J. Mol. Catal. A: Chem. 2015, 398, 407.

[10] Sellner. H.; Faber. C.; Rheiner. P. B.; Seebach. D. Chem.-Eur. J. 2000, 6, 3692.

[11] Fleischmann. C.; Ritter, H. Polym Int. 2015, 64, 724.

[12] Zhang, X.; Yang, N. F. Chin. J. Org. Chem. 2017, 37, 1027 (in Chinese).

(张勋，阳年发，有机化学, 2017, 37, 1027.)

[13] Wang, B.; Wang, S.; Xia, C.; Sun, W. Chem.-Eur. J. 2012. 18, 7332 .

[14] Lv, J.; Wang, X.; Liu, J.; Zhang, L.; Wang, Y. Tetrahedron: Asymmetry 2006, 17, 330.

[15] Yoo, M. S.; Kim, D. G.; Ha, M. W.; Jew, S. S.; Park, H. G.; Jeong, B. S. Tetrahedron Lett. 2010, 51, 5601. 